\title{
Aspects of the delayed alternation deficit produced by electrical stimulation in monkeys'
}

CHARLES G. GROSS

HARVARD UNIVERSITY

\begin{abstract}
Both intermittent and continuous electrical stimulation of lateral frontal cortex severely impaired delayed alternation performance of overtrained monkeys when tested in a Wisconsin General Test Apparatus. However, a similar effect was not obtained in an automatic delayed alternation apparatus.
\end{abstract}

Introduction

Several studies have shown that electrical stimulation of lateral frontal cortex can severely interfere with performance of specific tasks by monkeys. (Stamm, 1961; Weiskrantz, Mihailovic, \& Gross, 1960, 1962; Briese \& Olds, 1964; Cianci, 1965). Two of these studies differed in their methods and conclusions. Stamm, using an automatic apparatus, found stimulation of frontal cortex to impair acquisition but not retention of delayed alternation. He suggested that stimulation, unlike ablation of frontal cortex, disrupts "consolidation" or "the learning process" but does not affect the "engram" or "memory for behavior patterns." However, Weiskrantz et al, using a Wisconsin General Test Apparatus (WGTA) and different stimulus parameters found stimulation, like ablation, of frontal cortex to impair performance of delayed alternation in extensively overtrained monkeys.

The purpose of this investigation was to clarify the contribution of stimulus parameters (Experiment 1) and apparatus (Experiment 2) to the apparent discrepancies between these studies and thus to analyze further the differences between stimulation and ablation of frontal cortex.

\section{Subjects}

Four immature rhesus monkeys were implanted with Delgado-type cortical plate electrodes as described by Stamm (1961) and Weiskrantz et al (1962). Three pairs of electrodes were placed, bilaterally, on the banks of sulcus principalis. These Ss had previously been used in a study of cortical potentials during learning (Gerstein \& Gross, in preparation).

\section{Experiment I.}

Effect of Stimulus Parameters During WGTA Testing

Weiskrantz et al had stimulated $1 \mathrm{sec}$. out of every $4 \mathrm{sec}$. with $0.2 \mathrm{~ms}, 100 \mathrm{cps}$ pulses of a constant voltage set just below the motor threshold, whereas Stamm (1961) had stimulated continuously with $2 \mathrm{~ms} ., 22 \mathrm{cps}$ pulses, the voltage of which increased during the test session but remained below the motor threshold. This experiment examined effects of continuous and interrupted stimulation on delayed alternation performance in a WGTA.

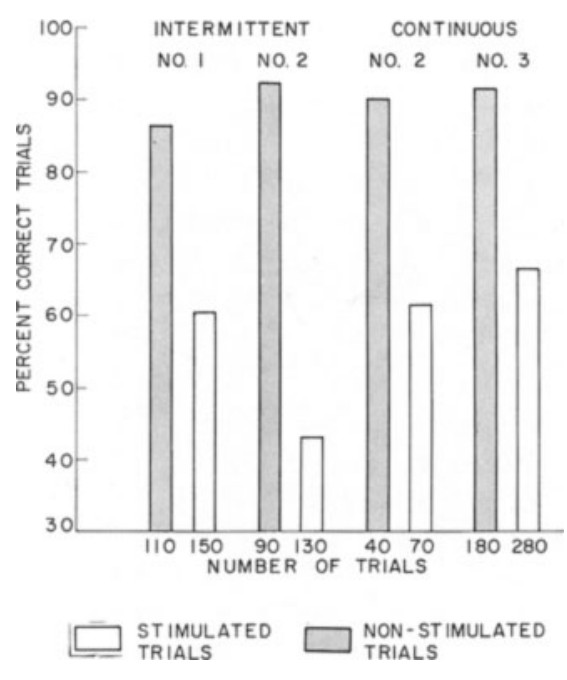

Fig. 1. Performance on delayed alternation in a WGTA during stimulation and without stimulation of monkeys No. 1,2, and 3 . "Intermittent" and "continuous" refer to the stimulation condition.

After reaching a criterion of 90 correct responses in 100 trials of $5 \mathrm{sec}$. delayed alternation, three monkeys were stimulated during delayed alternation performance with monophasic rectangular pulses, $2 \mathrm{~ms}$ in duration and at 22 cps. One monkey was stimulated for $1 \mathrm{sec}$. every $2 \mathrm{sec}$. (int $\epsilon$ r.mittent condition); the second monkey was stimulated continuously; and the third monkey was stimulated on different days with each of these procedures. In every session blocks of 10 control trials preceded, followed, and were distributed among blocks of 10 experimental (stimulation) trials.

At the start of each session the threshold for eliciting a motor response (head turning) was determined. The initial voltage applied during testing was $90 \%$ of this threshold voltage. During intermittent stimulation this voltage was kept constant throughout the test session and, in fact, remained constant for each monkey throughout the experiment. As may be seen in Fig. 1, this procedure produced a marked impairment on delayed alternation. In the continuous stimulation condition, when the voltage was kept constant, Ss made 3-6 errors in the first 10 experimental trials in each session but then would gradually improve to control levels within 10-20 trials. Furthermore, the motor threshold would increase $2-10$ volts above its initial value after $10-40$ trials of continuous stimulation. However, if the voltage 
was gradually increased (but kept subthreshold), the monkey's performance remained severely impaired throughout the period of stimulation (see Fig. 1). For all conditions, stimulation between a single pair of points had the same effects as stimulation between all four pairs. Neither continuous nor intermittent stimulation had any effect on retention of visual discriminations, thus demonstrating again the specificity of the deficit.

In summary, stimulation of frontal cortex, even with Stamm's parameters, severely impaired performance of a well-established delayed alternation habit in a WGTA. This result argues against Stamm's suggestion that such stimulation affects learning but not memory. Furthermore, interrupted stimulation was as effective as continuous stimulation, confirming Briese \& Old's (1964) belief that the aftereffects of stimulation may be "more disruptive than effects during stimulation itself." Experiment 2.

Effect of Stimulation During Automatic Testing

This experiment aimed at replicating Stamm's finding that, in an automatic apparatus, stimulation of frontal cortex does not impair retention of delayed alternation.

One monkey was trained to perform 5 sec. delayed alternation in an automatic apparatus to a criterion of 90 correct in 100 trials. As in Stamm's apparatus no screen was interposed between $S$ and manipulanda. Initiation of the trial was signalled by illumination of the two response buttons. Responses in the intertrial interval had previously been extinguished. In every test session blocks of 22 control trials alternated with blocks of 22 stimulation trials. The parameters of stimulation were the same as those that produced deficits in Experiment 1.

As long as performance on control trials remained at $15 \%$ errors or less, stimulation had no effect. However, if errors on control trials were increased (by increasing the delay, by mixing different delays in a single block of trials, by not running $S$ for several days, and by combinations of these procedures), then stimulation caused a further increase in errors. For example, in a delayed alternation session in which control performance was good (6.8\% errors), stimulation had no effect (4.6\% errors) whereas in a subsequent one in which control performance was poorer (22.7\% errors), stimulation had a significantly deleterious effect $\mathbf{3 3 . 2} \%$ errors)。

These results confirm Stamm's findings, using an automatic apparatus, of a positive relationship between level of control performance and degree of stimulationproduced deficit; stimulation had no effect when control performance was $85 \%$ accurate or better.

\section{Concluding Discussion}

In Experiment 1, frontal cortex stimulation impaired a well-established delayed alternation habit in a WGTA; ablation of frontal cortex is known to produce the same effect. In Experiment 2, frontal cortex stimulation had little or no effect on performance; similarly, frontal lesions have been reported to have a smaller effect on delayed alternation in an automatic apparatus than in a WGTA. (Stamm \& Pribram, 1960) or no effect at all (Battig, Rosvold, \& Mishkin, 1960)。Thus stimulation of frontal cortex can mimic, in a reversible fashion, ablation of this tissue.

The bases for the interaction between frontal stimulation or ablation and test apparatus remain to be analyzed. However, it should be noted that the automatic apparatus employed have several features that, when introduced in WGTA testing, have been shown to reduce the frontal deficit on delayed alternation. Among these features are absence of a screen interposed between $S$ and manipulanda, reduced illumination and reduction of gross bodily movement (Akert \& Warren, 1964).

The present study confirms and extends the previous results of Stamm (1961) and Weiskrantz, Mihailovic, \& Gross $(1960,1962)$. However, the finding that frontal stimulation disrupted an overtrained alternation habit in a WGTA and also, under certain conditions, in an automatic apparatus seems incompatible with the view that such stimulation affects the "learning" but not the "memory" of this habit.

\section{References}

Battig, K., Rosvold, H. E., \& Mishkin, M. Comparison of the effects of frontal and caudate lesions on delayed response and delayed alternation in monkeys. J. comp. physiol. Psychol., $1960,55,458-463$.

Briese, E., \& Olds, J. Reinforcing brain stimulation and memory in monkeys. Exp. Neurol., 1964, 10, 493-508.

Cianci, S. N. Effects of cortical and subcortical stimulation in delayed response in monkeys. Exp. Neurol., 1965, 11, 104-114.

Stamm, J. S. Electrical stimulation of frontal cortex in monkeys during learning of an alternation task. J. Neurophysiol., 1961, $24,414-426$.

Stamm, J. S., \& Pribaum, K. H. Effects of epileptogenic lesions in frontal cortex on learning and retention in monkeys. J. Neurophysiol., 1960, 23, 552-563.

Warren, J. M., \& Akert, L. (Eds.), The frontal granular cortex and behavior. New York: McGraw-Hill, 1964.

Weiskrantz, L., Mihailovic, L., \& Gross, C. G. Stimulation of frontal cortex and delayed alternation performance in the monkey. Science, 1960, 131, 1443-1444.

Weiskrantz, L., Mihailovic, L., \& Gross, C. G. Effects of stimulation of frontal cortex and hippocampus on behaviour in the monkey. Brain, 1962, 85, 487-504.

Note

1. These experiments were carried out at the Massachusetts Institute of Technology. Support was received from NIH Grants HD01079 and HD-01907 and NASA Grant NsG-496. The assistance of Miss E. Norris is very gratefully acknowledged. 